\title{
Existence of multiple solutions for a quasilinear Neumann problem with critical exponent
}

\author{
Yuanxiao Li $i^{*}$ and Suxia Xia
}

\section{"Correspondence:} yxiaoli06@163.com College of Science, Henan University of Technology,

Zhengzhou, P.R. China

\begin{abstract}
The main purpose of this paper is to establish the existence and multiplicity of nontrivial solutions for a quasilinear Neumann problem with critical exponent. It is shown, by the methods of the Lions concentration-compactness principle and the mountain pass lemma, that under certain conditions, the existence of nontrivial solutions are obtained.
\end{abstract}

MSC: 35B33; 35J50; 35J62; 35J92

Keywords: Quasilinear elliptic equation; Nontrivial solution; Neumann boundary condition; Critical Sobolev exponent; Lions concentration-compactness principle

\section{Introduction}

In this paper, we consider the following quasilinear elliptic problem with critical Sobolev exponent:

$$
\begin{cases}-\varepsilon^{p} \Delta_{p} u+V(x)|u|^{p-2} u=Q(x)|u|^{p^{*}-2} u+P(x)|u|^{q-2} u, & x \in \Omega, \\ |\nabla u|^{p-2} \frac{\partial u}{\partial v}=0, & x \in \partial \Omega\end{cases}
$$

where $\Omega \subset R^{N}$ is a bounded domain with smooth boundary, $\Delta_{p} u=\operatorname{div}\left(|\nabla u|^{p-2} \nabla u\right), \varepsilon>0$, $1<p<N, p<q<p^{*}=\frac{N p}{N-p}, v$ denotes the unit outward normal vector with respect to $\partial \Omega$. The weight functions $V(x), Q(x)$ and $P(x)$ are continuous on $\Omega$. Such problems arise in the theory of quasiregular and quasiconformal mapping or in the study of non-Newtonian fluids. In the latter case, the $p$ is a characteristic of the medium. Media with $p>2$ are called dilatant fluids and those with $p<2$ are called pseudoplastics. If $p=2$, they are Newtonian fluids.

The early study of Laplacian elliptic equation with critical Sobolev exponent was Pohozaev [1], the author established the nonexistence of nontrivial solution to the Dirichlet problems when $\Omega$ is a star-shaped domain with respect to the origin. Later, Brézis and Nirenberg [2] showed the existence of positive solutions by introducing the low-order perturbation terms, and Struwe [3] also obtained the global compactness result. Since then, the study of these elliptic problems with critical growth terms have been paid wide attentions in recent years (see [4-7]). Set $p=2, \varepsilon=1, P(x)=0, V(x)=\lambda$, then Problem (1.1)

(c) The Author(s) 2018. This article is distributed under the terms of the Creative Commons Attribution 4.0 International License (http://creativecommons.org/licenses/by/4.0/), which permits unrestricted use, distribution, and reproduction in any medium, provided you give appropriate credit to the original author(s) and the source, provide a link to the Creative Commons license, and indicate if changes were made. 
reduces to the following semilinear elliptic problem:

$$
\begin{cases}-\Delta u+\lambda u=Q(x)|u|^{2^{*}-2} u, & x \in \Omega \\ \frac{\partial u}{\partial \nu}=0, & x \in \Omega .\end{cases}
$$

Comte and Knaap [8] proved that there exists a nontrivial solution of problem (1.2) by variational method if $Q(x)=1$ and $\lambda=-\mu$. Chabrowski and Willem [9] studied this problem with the assumption that the function $Q(x)$ is nonnegative and Hölder continuous, they obtained the existence of least energy solutions by solving minimization problem corresponding to

$$
S_{\lambda}=\inf _{u \in H^{1}(\Omega), \int_{\Omega} Q(x)|u|^{2^{*}} \mathrm{~d} x \neq 0} \frac{\int_{\Omega}\left(|\nabla u|^{2}+\lambda u^{2}\right) \mathrm{d} x}{\left(\int_{\Omega} Q(x)|u|^{2^{*}} \mathrm{~d} x\right)^{\frac{2}{2^{*}}}} .
$$

Subsequently, Chabrowski and Girão [10] investigated the existence and nonexistence of least energy solutions when the function $Q(x)$ has some symmetry properties. For more relevant information as regards the corresponding problems, the interested reader may refer to [11-21] and the references therein.

As for quasilinear elliptic problems with critical Sobolev exponent, the existence and multiplicity of solutions have also been studied extensively. Abreu et al. [22] studied the following nonhomogeneous Neumann boundary problems:

$$
\begin{cases}-\Delta_{p} u+\lambda u^{p-1}=u^{q}, & x \in \Omega, \\ u>0, & x \in \Omega, \\ |\nabla u|^{p-2} \frac{\partial u}{\partial \nu}=\varphi, & x \in \partial \Omega,\end{cases}
$$

where $p-1<q \leq p^{*}-1, \varphi \in C^{\alpha}(\bar{\Omega}), 0<\alpha<1, \varphi \not \equiv 0$. They proved that there exists a $\lambda^{*}>0$ such that problem (1.3) has at least two positive solutions if $\lambda>\lambda^{*}$, has at least one positive solution if $\lambda=\lambda^{*}$ and has no positive solution if $\lambda<\lambda^{*}$ relying on the lower and upper solutions method and variational approach. Zhao et al. [23] discussed the quasilinear elliptic problem of the form

$$
\begin{cases}-\Delta_{p} u+\lambda(x)|u|^{p-2} u=|u|^{p^{*}-2} u+|u|^{r-2} u, & x \in \Omega, \\ |\nabla u|^{p-2} \frac{\partial u}{\partial v}=\eta|u|^{p-2} u, & x \in \partial \Omega\end{cases}
$$

they showed that there exists at least a nontrivial solution when $p<r<p^{*}$ and there exist infinitely many solutions when $1<r<p$ by using the Mountain pass theorem and the concentration-compactness principle. Some authors also studied the critical Sobolev exponent for quasilinear equations and the corresponding evolution problems with Neumann boundary conditions, the reader may also refer to [24-37].

Motivated by the results of the above papers, we discuss the existence of nontrivial nonnegative solutions to Problem (1.1) by a variational method. The special features of this problem are the following. Firstly, due to the lack of compactness of the embedding of $W^{1, p}(\Omega) \hookrightarrow L^{p^{*}}(\Omega)$, we cannot use the standard variational argument directly. In order to overcome this difficulty and obtain the existence of solutions, we have to add restrictions 
on the weight functions $Q(x)$ and $P(x)$ to prove the corresponding functional of Problem $(1.1)$ satisfies $(P S)_{c}$-condition in a suitable range by the Lions concentration-compactness principle. Secondly, the weight function $V(x)$ may be unbounded near the boundary $\partial \Omega$, which leads to the space $W^{1, p}(\Omega)$ is not suitable for our problem. To solve such problem, we have to introduce a suitable weighted Sobolev space.

For the sake of convenience, we introducing a new parameter $\lambda=\varepsilon^{-p}$, then Problem (1.1) may be rewritten as the following problem:

$$
\begin{cases}-\Delta_{p} u+\lambda V(x)|u|^{p-2} u=\lambda Q(x)|u|^{p^{*}-2} u+\lambda P(x)|u|^{q-2} u, & x \in \Omega, \\ |\nabla u|^{p-2} \frac{\partial u}{\partial v}=0, & x \in \partial \Omega .\end{cases}
$$

Throughout this paper, we make some assumptions on the weight functions $Q(x), P(x)$, $V(x)$ as the following:

(A1) $Q(x), P(x)$ are continuous on $\bar{\Omega}$, and $Q(x)>0, P(x) \geq 0$ for $x \in \bar{\Omega}$;

(A2) $V(x)$ is continuous in $\Omega$, and $V(x) \geq 0, V(x) \not \equiv$ for $x \in \Omega$.

Set $Q_{m}=\max _{x \in \partial \Omega} Q(x), Q_{M}=\max _{x \in \bar{\Omega}} Q(x), P_{M}=\max _{x \in \bar{\Omega}} P(x)$. The main results of this paper are the following.

Theorem 1.1 Suppose that (A1), (A2) hold, $H(0)>0$ and $Q_{m}=Q(0)$. If functions $Q(x)$, $V(x)$ satisfy

(A3) $Q_{M} \leq 2^{\frac{p}{N-p}} Q_{m}$, and $|Q(x)-Q(0)|=o\left(|x|^{\alpha}\right)$ as $x \rightarrow 0$, where $1<\alpha<\frac{N}{p-1}$;

(A4) $\int_{\Omega \cap B(0, \delta)} V^{r^{\prime}} \mathrm{d} x<\infty$, where $\frac{1}{r}+\frac{1}{r^{\prime}}=1,1<r<\frac{N(p-1)}{N p+2 p-N-p^{2}-1}, \delta>0$.

Then Problem (1.5) has at least one nontrivial solution for every $\lambda>0$ and $N \geq 2 p$, where $H(0)$ will be later determined.

Theorem 1.2 Suppose that (A1), (A2) hold. If $Q_{M}>2^{\frac{p}{N-p}} Q_{m}$ and functions $P(x), V(x)$ satisfy

(A5) $P(x) \not \equiv 0$ for $x \in \Omega$, and $V \in L^{1}(\Omega)$.

Then there exists a $\lambda_{*}>0$ such that Problem (1.5) has at least one nontrivial solution for $0<\lambda<\lambda_{*}$.

Theorem 1.3 Suppose that (A1), (A2) hold. If $Q_{M}>2^{\frac{p}{N-p}} Q_{m}$ and functions $P(x), V(x)$ satisfy

(A6) $P(x)>0$ for $x \in \bar{\Omega}$;

(A7) there exist $x_{0} \in \Omega$ and constant $\delta>0$ such that $V(x)=0$ for $x \in B\left(x_{0}, \delta\right) \subset \Omega$.

Then there exists $a \lambda^{*}>0$ such that Problem (1.5) has at least one nontrivial solution for $\lambda>\lambda^{*}$.

Theorem 1.4 Suppose that (A1), (A2) hold. If $Q_{M}>2^{\frac{p}{N-p}} Q_{m}$ and functions $P(x), V(x)$ satisfy the conditions (A6) and (A7). Then, for every integer $n$, there exists a constant $\Lambda_{n}>0$ such that Problem (1.5) has at least $n$ pairs of nontrivial solutions for $\lambda>\Lambda_{n}$.

\section{Preliminaries}

Firstly, we define the weighted Sobolev space

$$
W_{\lambda, V}^{1, p}(\Omega)=\left\{u ; D_{i} u \in L^{p}(\Omega), i=1,2, \ldots, N, \int_{\Omega} V(x)|u|^{p} \mathrm{~d} x<+\infty\right\}
$$


with norm $\|u\|_{\lambda, V}=\left(\int_{\Omega}\left(|\nabla u|^{p}+\lambda V(x)|u|^{p}\right) \mathrm{d} x\right)^{\frac{1}{p}}$. Obviously, norms $\|u\|_{\lambda, V}$ and $\|u\|_{V}$ are equivalent, $W_{\lambda, V}^{1, p}(\Omega) \hookrightarrow W^{1, p}(\Omega)$, where $\|u\|_{V}=\left(\int_{\Omega}\left(|\nabla u|^{p}+V(x)|u|^{p}\right) \mathrm{d} x\right)^{\frac{1}{p}}$.

By using the Sobolev embedding theorem, we know that there exists a constant $C_{q}>0$ such that

$$
|u|_{q} \leq C_{q}\|u\|_{V} \leq C_{q}\|u\|_{\lambda V}, \quad \text { for } \lambda \geq 1, u \in W_{\lambda, V}^{1, p}(\Omega)
$$

and

$$
|u|_{q} \leq C_{q}\|u\|_{V} \leq \lambda^{-\frac{1}{p}} C_{q}\|u\|_{\lambda V}, \quad \text { for } 0<\lambda<1, u \in W_{\lambda, V}^{1, p}(\Omega)
$$

where $|u|_{q}=\left(\int_{\Omega}|u|^{q} \mathrm{~d} x\right)^{\frac{1}{q}}, q \in\left(p, p^{*}\right)$.

Next, we give the definition of weak solution to Problem (1.5).

Definition 2.1 A function $u \in W_{\lambda, V}^{1, p}(\Omega)$ is said to be a weak solution of Problem (1.5) if it satisfies

$$
\begin{aligned}
& \int_{\Omega}|\nabla u|^{p-2} \nabla u \nabla \psi \mathrm{d} x+\lambda \int_{\Omega} V(x)|u|^{p-2} u \psi \mathrm{d} x \\
& \quad=\lambda \int_{\Omega} Q(x)|u|^{p^{*}-2} u \psi \mathrm{d} x+\lambda \int_{\Omega} P(x)|u|^{q-2} u \psi \mathrm{d} x, \quad \forall \psi \in W_{\lambda, V}^{1, p}(\Omega) .
\end{aligned}
$$

Thus, the corresponding energy functional of Problem (1.5) is defined in $W_{\lambda, V}^{1, p}(\Omega)$ by

$$
J_{\lambda}(u)=\frac{1}{p} \int_{\Omega}\left(|\nabla u|^{p}+\lambda V(x)|u|^{p}\right) \mathrm{d} x-\frac{\lambda}{p^{*}} \int_{\Omega} Q(x)|u|^{p^{*}} \mathrm{~d} x-\frac{\lambda}{q} \int_{\Omega} P(x)|u|^{q} \mathrm{~d} x .
$$

Let $S$ be the best Sobolev constants, namely

$$
S=\inf _{D^{1, p}\left(R^{N}\right) \backslash\{0\}} \frac{\int_{\Omega}|\nabla u|^{p} \mathrm{~d} x}{\left(\int_{\Omega}|u|^{p^{*}} \mathrm{~d} x\right)^{\frac{p}{p^{*}}}},
$$

where $D^{1, p}\left(R^{N}\right)=\left\{u \in L^{p^{*}}\left(R^{N}\right):|\nabla u| \in L^{p}\left(R^{N}\right)\right\}$. This constant $S$ is achieved by the functional $u_{\varepsilon}$ given by

$$
u_{\varepsilon}(x)=C_{N p} \varepsilon^{\frac{N-p}{p^{2}}}\left(\varepsilon+|x|^{\frac{p}{p-1}}\right)^{\frac{p-N}{p}}
$$

where the constant $C_{N p}$ is chosen such that $-\Delta_{p} u_{\varepsilon}=\left|u_{\varepsilon}\right|^{p^{*}-1}$ in $R^{N}$ (see [22] for details).

In order to obtain the existence of solutions to Problem (1.5), we need the following lemma.

Lemma 2.1 For each $\lambda>0$,

(i) there exist constants $\beta_{\lambda}, \rho_{\lambda}>0$ such that $J_{\lambda}(u) \geq \beta_{\lambda}$ for $\|u\|_{\lambda V}=\rho_{\lambda}$;

(ii) there exists an $u_{0} \in W_{\lambda, V}^{1, p}(\Omega)$ with $u_{0} \not \equiv 0$ such that $J_{\lambda}\left(u_{0}\right)<0$ for $\left\|u_{0}\right\|_{\lambda V}>\rho_{\lambda}$. 
Proof (i) Firstly, we consider the case $\lambda \geq 1$. let $\|u\|_{V}^{p}=\rho^{p}$, then $\rho_{\lambda}^{p}=\|u\|_{\lambda V}^{p} \leq \lambda \rho^{p}$. Using (2.1) and (2.3), we have

$$
\begin{aligned}
J_{\lambda}(u) & \geq \frac{1}{p}\|u\|_{\lambda V}^{p}-\frac{\lambda}{p^{*}} Q_{M} S^{p^{\frac{p^{*}}{p}}}\left(\int_{\Omega}|\nabla u|^{p} \mathrm{~d} x\right)^{\frac{p^{*}}{p}}-\frac{\lambda}{q} P_{M} C_{q}^{q}\|u\|_{\lambda V}^{q} \\
& \geq \frac{1}{p}\|u\|_{\lambda V}^{p}-\frac{\lambda}{p^{*}} Q_{M} S^{p^{\frac{p^{*}}{p}}}\|u\|_{\lambda V}^{p^{*}}-\frac{\lambda}{q} P_{M} C_{q}^{q}\|u\|_{\lambda V}^{q} \\
& \geq \rho_{\lambda}^{p}\left(\frac{1}{p}-\frac{\lambda^{\frac{p^{*}}{p}}}{p^{*}} Q_{M} S^{-\frac{p^{*}}{p}} \rho^{p^{*}-p}-\frac{\lambda^{\frac{q}{p}}}{q} P_{M} C_{q}^{q} \rho^{q-p}\right) .
\end{aligned}
$$

Since $p<q<p^{*}$, taking $\rho>0$ small enough, there exists a $\beta_{\lambda}>0$ such that $J_{\lambda}(u) \geq \beta_{\lambda}$ for $\|u\|_{\lambda V}=\rho_{\lambda}$.

If $0<\lambda<1$, let $\|u\|_{V}^{p}=\rho^{p}$, then $\rho>\rho_{\lambda}>\lambda^{\frac{1}{p}} \rho$. Combining (2.2) with (2.3), we see that

$$
\begin{aligned}
J_{\lambda}(u) & \geq \frac{1}{p}\|u\|_{\lambda V}^{p}-\frac{\lambda}{p^{*}} Q_{M} S^{\frac{p^{*}}{p}}\|u\|_{V}^{p^{*}}-\frac{\lambda}{q} P_{M} C_{q}^{q}\|u\|_{V}^{q} \\
& >\lambda \rho^{p}\left(\frac{1}{p}-\frac{1}{p^{*}} Q_{M} S^{-\frac{p^{*}}{p}} \rho^{p^{*}-p}-\frac{1}{q} P_{M} C_{q}^{q} \rho^{q-p}\right) .
\end{aligned}
$$

Since $p<q<p^{*}$, taking $\rho>0$ small enough, there exists a $\beta_{\lambda}>0$ such that $J_{\lambda}(u) \geq \beta_{\lambda}$ for $\|u\|_{\lambda V}=\rho_{\lambda}$.

(ii) For $u \in W_{\lambda, V}^{1, p}(\Omega)$ and $u \not \equiv 0$, we define

$$
J_{\lambda}(t u)=\frac{t^{p}}{p}\|u\|_{\lambda V}^{p}-\frac{t^{p^{*}}}{p^{*}} \lambda \int_{\Omega} Q(x)|u|^{p^{*}} \mathrm{~d} x-\frac{t^{q}}{q} \lambda \int_{\Omega} P(x)|u|^{q} \mathrm{~d} x, \quad t>0,
$$

it follows from $\lim _{t \rightarrow+\infty} J_{\lambda}(t u)=-\infty$ that there exists a $t_{0}>0$ such that $\left\|t_{0} u\right\|_{\lambda V}>\rho_{\lambda}$ and $J_{\lambda}\left(t_{0} u\right)<0$. Letting $u_{0}=t_{0} u$, then condition (ii) holds. The proof of Lemma 2.1 is completed.

Define

$$
c=\inf _{h \in \Gamma} \sup _{t \in[0,1]} J_{\lambda}(h(t)),
$$

where $\Gamma=\left\{h \in C\left([0,1], W_{\lambda, V}^{1, p}(\Omega)\right) \mid h(0)=0, h(1)=t_{0} u=u_{0}\right\}$. Using Lemma 2.1, we know that the energy functional $J_{\lambda}(u)$ satisfies the geometry of the mountain pass lemma, then there exists a $(P S)_{c}$-sequence $\left\{u_{n}\right\} \subset W_{\lambda, V}^{1, p}(\Omega)$ such that $J_{\lambda}\left(u_{n}\right) \rightarrow c, J_{\lambda}^{\prime}\left(u_{n}\right) \rightarrow 0$ as $n \rightarrow \infty$.

Lemma 2.2 Assume (A1), (A2) hold, and $\left\{u_{n}\right\}$ be a $(P S)_{c}$-sequence at the level of $c$ for $J_{\lambda}$ with $c<c^{*}=\min \left\{\frac{S^{\frac{N}{p}}}{N \lambda \frac{N-p}{p} Q_{M}^{\frac{N-p}{p}}}, \frac{S^{\frac{N}{p}}}{2 N \lambda^{\frac{N-p}{p}} Q_{m}^{\frac{N-p}{p}}}\right\}$, then $\left\{u_{n}\right\}$ is relatively compact in $W_{\lambda, V}^{1, p}(\Omega)$.

Proof Firstly, we prove that $\left\{u_{n}\right\}$ is bounded. Since $J_{\lambda}\left(u_{n}\right) \rightarrow c, J_{\lambda}^{\prime}\left(u_{n}\right) \rightarrow 0$ as $n \rightarrow \infty$, we have

$$
\begin{aligned}
J_{\lambda}\left(u_{n}\right) & =\frac{1}{p} \int_{\Omega}\left(\left|\nabla u_{n}\right|^{p}+\lambda V(x)\left|u_{n}\right|^{p}\right) \mathrm{d} x-\frac{\lambda}{p^{*}} \int_{\Omega} Q(x)\left|u_{n}\right|^{p^{*}} \mathrm{~d} x-\frac{\lambda}{q} \int_{\Omega} P(x)\left|u_{n}\right|^{q} \mathrm{~d} x \\
& =c+o(1)\left\|u_{n}\right\|,
\end{aligned}
$$




$$
\begin{aligned}
& \int_{\Omega}\left(\left|\nabla u_{n}\right|^{p}+\lambda V(x)\left|u_{n}\right|^{p}\right) \mathrm{d} x-\lambda \int_{\Omega} Q(x)\left|u_{n}\right|^{p^{*}} \mathrm{~d} x-\lambda \int_{\Omega} P(x)\left|u_{n}\right|^{q} \mathrm{~d} x \\
& \quad=o(1)\left\|u_{n}\right\| .
\end{aligned}
$$

Combining (A1) and (A2), one has

$$
\begin{aligned}
c+o(1)\left\|u_{n}\right\| & =\left(\frac{1}{p}-\frac{1}{q}\right) \int_{\Omega}\left(\left|\nabla u_{n}\right|^{p}+\lambda V(x)\left|u_{n}\right|^{p}\right) \mathrm{d} x+\lambda\left(\frac{1}{q}-\frac{1}{p^{*}}\right) \int_{\Omega} Q(x)\left|u_{n}\right|^{p^{*}} \mathrm{~d} x \\
& \geq\left(\frac{1}{p}-\frac{1}{q}\right)\left\|u_{n}\right\|_{\lambda V}^{p} .
\end{aligned}
$$

Thus, we can find that $\left\{u_{n}\right\}$ is bounded in $W_{\lambda, V}^{1, p}(\Omega)$.

Next, we prove that $\left\{u_{n}\right\}$ is relatively compact in $W_{\lambda, V}^{1, p}(\Omega)$. Since $\left\{u_{n}\right\}$ is bounded in $W_{\lambda, V}^{1, p}(\Omega)$, there exists a subsequence, still denoted by $\left\{u_{n}\right\}$ and $u \in W_{\lambda, V}^{1, p}(\Omega)$ such that

$$
\begin{array}{ll}
u_{n} \rightarrow u & \text { weakly in } W_{\lambda, V}^{1, p}(\Omega), \\
u_{n} \rightarrow u & \text { weakly in } L^{p^{*}}(\Omega), \\
u_{n} \rightarrow u & \text { strongly in } L^{q}(\Omega), p \leq q<p^{*}, \\
u_{n} \rightarrow u & \text { a.e. in } \Omega .
\end{array}
$$

By the Lions concentration-compactness principle [38], there exists at most set $J$, a set of different points $\left\{x_{j}\right\}_{j \in J} \subset \bar{\Omega}$, sets of nonnegative real numbers $\left\{\mu_{j}\right\}_{j \in J},\left\{v_{j}\right\}_{j \in J}$ such that

$$
\begin{aligned}
& \left|\nabla u_{n}\right|^{p}-\mathrm{d} \mu \geq|\nabla u|^{p}+\sum_{j \in J} \mu_{j} \delta_{x_{j}}, \\
& \left|u_{n}\right|^{p^{*}} \rightarrow \mathrm{d} v=|u|^{p^{*}}+\sum_{j \in J} v_{j} \delta_{x_{j}},
\end{aligned}
$$

where $\delta_{x}$ is the Dirac mass at $x$, and the constants $\mu_{j}, v_{j}$ satisfying

$$
\begin{aligned}
& S v_{j}^{\frac{p}{p^{*}}} \leq \mu_{j}, \quad \text { where } x_{j} \in \Omega, \\
& \frac{S}{2^{\frac{p}{N}}} v_{j}^{\frac{p}{p^{*}}} \leq \mu_{j}, \quad \text { where } x_{j} \in \partial \Omega .
\end{aligned}
$$

Next, we prove $\mu_{j}=0$ and $v_{j}=0$, where $j \in J$. In fact, choosing $\varepsilon>0$ sufficiently small such that $B_{\varepsilon}\left(x_{i}\right) \cap B_{\varepsilon}\left(x_{j}\right)=\varnothing$ for $i \neq j, i, j \in J$. Let $\phi_{\varepsilon}^{j}(x)$ be a smooth cut off function centered at $x_{j}$ such that

$$
0 \leq \phi_{\varepsilon}^{j}(x) \leq 1 \quad \text { for }\left|x-x_{j}\right|<\varepsilon, \quad \phi_{\varepsilon}^{j}(x)=\left\{\begin{array}{l}
1,\left|x-x_{j}\right| \leq \frac{\varepsilon}{2}, \\
0,\left|x-x_{j}\right| \geq \varepsilon,
\end{array} \quad \text { and } \quad\left|\nabla \phi_{\varepsilon}^{j}\right| \leq \frac{4}{\varepsilon} .\right.
$$

Noting that

$$
\begin{aligned}
& \left\langle J_{\lambda}^{\prime}\left(u_{n}\right), u_{n} \phi_{\varepsilon}^{j}(x)\right\rangle \\
& \quad=\int_{\Omega}\left|\nabla u_{n}\right|^{p} \phi_{\varepsilon}^{j}(x) \mathrm{d} x+\int_{\Omega}\left|\nabla u_{n}\right|^{p-2} \nabla u_{n} \nabla \phi_{\varepsilon}^{j}(x) u_{n} \mathrm{~d} x
\end{aligned}
$$




$$
\begin{aligned}
& +\lambda \int_{\Omega} V(x)\left|u_{n}\right|^{p} \phi_{\varepsilon}^{j}(x) \mathrm{d} x-\lambda \int_{\Omega} Q(x)\left|u_{n}\right|^{p^{*}} \phi_{\varepsilon}^{j}(x) \mathrm{d} x \\
& -\lambda \int_{\Omega} P(x)\left|u_{n}\right|^{q} \phi_{\varepsilon}^{j}(x) \mathrm{d} x,
\end{aligned}
$$

and by (2.4), we have

$$
\begin{aligned}
& \lim _{\varepsilon \rightarrow 0} \lim _{n \rightarrow \infty} \int_{\Omega}\left|\nabla u_{n}\right|^{p} \phi_{\varepsilon}^{j}(x) \mathrm{d} x \geq \lim _{\varepsilon \rightarrow 0}\left[\int_{\Omega}|\nabla u|^{p} \phi_{\varepsilon}^{j}(x) \mathrm{d} x+\int_{\Omega} \sum_{j \in J} \mu_{j} \delta_{x_{j}} \phi_{\varepsilon}^{j}(x) \mathrm{d} x\right] \geq \mu_{j}, \\
& \lim _{\varepsilon \rightarrow 0} \lim _{n \rightarrow \infty} \int_{\Omega}\left|\nabla u_{n}\right|^{p-2} \nabla u_{n} \nabla \phi_{\varepsilon}^{j}(x) u_{n} \mathrm{~d} x=0, \\
& \lim _{\varepsilon \rightarrow 0} \lim _{n \rightarrow \infty} \int_{\Omega} V(x)\left|u_{n}\right|^{p} \phi_{\varepsilon}^{j}(x) \mathrm{d} x=0, \\
& \lim _{\varepsilon \rightarrow 0} \lim _{n \rightarrow \infty} \int_{\Omega} Q(x)\left|u_{n}\right|^{p^{*}} \phi_{\varepsilon}^{j}(x) \mathrm{d} x=Q\left(x_{j}\right) v_{j}, \\
& \lim _{\varepsilon \rightarrow 0} \lim _{n \rightarrow \infty} \int_{\Omega} P(x)\left|u_{n}\right|^{q} \phi_{\varepsilon}^{j}(x) \mathrm{d} x=0 .
\end{aligned}
$$

Thus,

$$
0=\lim _{\varepsilon \rightarrow 0} \lim _{n \rightarrow \infty}\left\langle J_{\lambda}^{\prime}\left(u_{n}\right), u_{n} \phi_{\varepsilon}^{j}(x)\right\rangle \geq \mu_{j}-\lambda Q\left(x_{j}\right) v_{j}
$$

If $v_{j} \neq 0$, by (2.5) and (2.6), we find that

$$
\begin{aligned}
& v_{j} \geq \frac{S^{\frac{N}{p}}}{\lambda^{\frac{N}{p}} Q^{\frac{N}{p}}\left(x_{j}\right)}, \quad x_{j} \in \Omega, \\
& v_{j} \geq \frac{S^{\frac{N}{p}}}{2 \lambda^{\frac{N}{p}} Q^{\frac{N}{p}}\left(x_{j}\right)}, \quad x_{j} \in \partial \Omega .
\end{aligned}
$$

On the other hand,

$$
\begin{aligned}
c & =\lim _{n \rightarrow \infty}\left(J_{\lambda}\left(u_{n}\right)-\frac{1}{p}\left\langle J_{\lambda}^{\prime}\left(u_{n}\right), u_{n}\right\rangle\right) \\
& =\left(\frac{1}{p}-\frac{1}{p^{*}}\right) \lambda \int_{\Omega} Q(x)|u|^{p^{*}} \mathrm{~d} x+\left(\frac{1}{p}-\frac{1}{q}\right) \lambda \int_{\Omega} P(x)|u|^{q} \mathrm{~d} x+\left(\frac{1}{p}-\frac{1}{p^{*}}\right) \lambda \sum_{j \in J} Q\left(x_{j}\right) v_{j} \\
& \geq \frac{1}{N} \lambda \sum_{j \in J} Q\left(x_{j}\right) v_{j}
\end{aligned}
$$

consequently,

$$
\begin{aligned}
& c \geq \frac{1}{N} \lambda Q\left(x_{j}\right) v_{j} \geq \frac{S^{\frac{N}{p}}}{N \lambda^{\frac{N-p}{p}} Q_{M}^{\frac{N-p}{p}}}, \quad x_{j} \in \Omega, \\
& c \geq \frac{1}{N} \lambda Q\left(x_{j}\right) v_{j} \geq \frac{S^{\frac{N}{p}}}{2 N \lambda^{\frac{N-p}{p}} Q_{m}^{\frac{N-p}{p}}}, \quad x_{j} \in \partial \Omega,
\end{aligned}
$$

which is a contradiction. Hence, $\mu_{j}=0, v_{j}=0$ and we find that $u_{n} \rightarrow u$ strongly in $L^{p^{*}}(\Omega)$. 
Now, we prove that $u_{n} \rightarrow u$ strongly in $W_{\lambda, V}^{1, p}(\Omega)$. We have

$$
\begin{aligned}
& \left\langle J_{\lambda}^{\prime}\left(u_{n}\right)-J_{\lambda}^{\prime}(u), u_{n}-u\right\rangle \\
& =\left\|u_{n}\right\|_{\lambda, V}^{p}+\|u\|_{\lambda, V}^{p}-\int_{\Omega}\left(\left|\nabla u_{n}\right|^{p-2} \nabla u_{n} \nabla u+\lambda V(x)\left|u_{n}\right|^{p-2} u_{n} u\right) \mathrm{d} x \\
& \quad-\int_{\Omega}\left(|\nabla u|^{p-2} \nabla u \nabla u_{n}+\lambda V(x)|u|^{p-2} u u_{n}\right) \mathrm{d} x-I-I I,
\end{aligned}
$$

where

$$
\begin{aligned}
& I=\lambda \int_{\Omega} Q(x)\left(\left|u_{n}\right|^{p^{*}-2} u_{n}-|u|^{p^{*}-2} u\right)\left(u_{n}-u\right) \mathrm{d} x, \\
& I I=\lambda \int_{\Omega} P(x)\left(\left|u_{n}\right|^{q-2} u_{n}-|u|^{q-2} u\right)\left(u_{n}-u\right) \mathrm{d} x .
\end{aligned}
$$

By the Hölder inequality and Jensen's inequality

$$
(a+b)^{\alpha}(c+d)^{1-\alpha} \geq a^{\alpha} c^{1-\alpha}+b^{\alpha} d^{1-\alpha},
$$

where $\alpha \in(0,1), a>0, b>0, c>0, d>0$, we have

$$
\begin{aligned}
& \int_{\Omega}\left(\left|\nabla u_{n}\right|^{p-2} \nabla u_{n} \nabla u+\lambda V(x)\left|u_{n}\right|^{p-2} u_{n} u\right) \mathrm{d} x \\
& \leq\left(\int_{\Omega}\left|\nabla u_{n}\right|^{p} \mathrm{~d} x\right)^{\frac{p-1}{p}}\left(\int_{\Omega}|\nabla u|^{p} \mathrm{~d} x\right)^{\frac{1}{p}} \\
& \quad+\left(\lambda \int_{\Omega} V(x)\left|u_{n}\right|^{p} \mathrm{~d} x\right)^{\frac{p-1}{p}}\left(\lambda \int_{\Omega} V(x)|u|^{p} \mathrm{~d} x\right)^{\frac{1}{p}} \\
& \leq\left(\int_{\Omega}\left|\nabla u_{n}\right|^{p}+\lambda V(x)\left|u_{n}\right|^{p} \mathrm{~d} x\right)^{\frac{p-1}{p}}\left(\int_{\Omega}|\nabla u|^{p}+\lambda V(x)|u|^{p} \mathrm{~d} x\right)^{\frac{1}{p}}=\left\|u_{n}\right\|_{\lambda V}^{p-1}\|u\|_{\lambda V} .
\end{aligned}
$$

Similarly, we get

$$
\begin{aligned}
\int_{\Omega}\left(|\nabla u|^{p-2} \nabla u \nabla u_{n}+\lambda V(x)|u|^{p-2} u u_{n}\right) \mathrm{d} x \leq\|u\|_{\lambda V}^{p-1}\left\|u_{n}\right\|_{\lambda V} \\
|I| \leq \lambda Q_{M}\left[\int_{\Omega}\left|u_{n}\right|^{p^{*}-1}\left|u_{n}-u\right| \mathrm{d} x+\int_{\Omega}|u|^{p^{*}-1}\left|u_{n}-u\right| \mathrm{d} x\right] \\
\leq \lambda Q_{M}\left(\int_{\Omega}\left|u_{n}\right|^{p^{*}} \mathrm{~d} x\right)^{\frac{p^{*}-1}{p^{*}}}\left(\int_{\Omega}\left|u_{n}-u\right|^{p^{*}} \mathrm{~d} x\right)^{\frac{1}{p^{*}}} \\
+\lambda Q_{M}\left(\int_{\Omega}|u|^{p^{*}} \mathrm{~d} x\right)^{\frac{p^{*}-1}{p^{*}}}\left(\int_{\Omega}\left|u_{n}-u\right|^{p^{*}} \mathrm{~d} x\right)^{\frac{1}{p^{*}}} \\
|I I| \leq \lambda P_{M}\left[\int_{\Omega}\left|u_{n}\right|^{q-1}\left|u_{n}-u\right| \mathrm{d} x+\int_{\Omega}|u|^{q-1}\left|u_{n}-u\right| \mathrm{d} x\right] \\
\leq \lambda P_{M}\left(\int_{\Omega}\left|u_{n}\right|^{q} \mathrm{~d} x\right)^{\frac{q-1}{q}}\left(\int_{\Omega}\left|u_{n}-u\right|^{q} \mathrm{~d} x\right)^{\frac{1}{q}} \\
+\lambda P_{M}\left(\int_{\Omega}|u|^{q} \mathrm{~d} x\right)^{\frac{q-1}{q}}\left(\int_{\Omega}\left|u_{n}-u\right|^{q} \mathrm{~d} x\right)^{\frac{1}{q}} \cdot
\end{aligned}
$$


We have

$$
0=\lim _{n \rightarrow \infty}\left\langle J_{\lambda}^{\prime}\left(u_{n}\right)-J_{\lambda}^{\prime}(u), u_{n}-u\right\rangle \geq \lim _{n \rightarrow \infty}\left(\left\|u_{n}\right\|_{\lambda V}^{p-1}-\|u\|_{\lambda V}^{p-1}\right)\left(\left\|u_{n}\right\|_{\lambda V}-\|u\|_{\lambda V}\right) \geq 0 .
$$

Hence, $u_{n} \rightarrow u$ strongly in $W_{\lambda, V}^{1, p}(\Omega)$.

Since $0 \in \partial \Omega$ and $\partial \Omega \in C^{2}$, the boundary $\partial \Omega$ near the origin can be represented $x_{N}=$ $h\left(x^{\prime}\right)=\frac{1}{2} \sum_{i=1}^{N-1} \lambda_{i} x_{i}^{2}+o\left(\left|x^{\prime}\right|^{2}\right)$, where $x^{\prime}=\left(x_{1}, x_{2}, \ldots, x_{N-1}\right) \in D(0, \delta)=B(0, \delta) \cap\left\{x_{N}=0\right\}, \lambda_{i}$ $(i=1,2, \ldots, N-1)$ are the principal curvatures of $\partial \Omega$ at 0 and the mean curvatures $H(0)=$ $\frac{1}{N-1} \sum_{i=1}^{N-1} \lambda_{i}>0$. Then the following lemma holds.

Lemma $2.3([22])$

(1) For $N>2 p-1$ and $\varepsilon>0$ small enough,

$$
\begin{aligned}
& \int_{\Omega}\left|\nabla u_{\varepsilon}\right|^{p} \mathrm{~d} x=\int_{R_{+}^{N}}\left|\nabla u_{\varepsilon}\right|^{p} \mathrm{~d} x-K_{1}(\varepsilon)+o\left(\varepsilon^{\frac{p-1}{p}}\right), \\
& \int_{\Omega}\left|u_{\varepsilon}\right|^{p^{*}} \mathrm{~d} x=\int_{R_{+}^{N}}\left|u_{\varepsilon}\right|^{p^{*}} \mathrm{~d} x-K_{2}(\varepsilon)+o\left(\varepsilon^{\frac{p-1}{p}}\right),
\end{aligned}
$$

where $K_{1}(\varepsilon), K_{2}(\varepsilon)$ satisfy

$$
\begin{aligned}
& \lim _{\varepsilon \rightarrow 0} \varepsilon^{-\frac{p-1}{p}} K_{1}(\varepsilon)=\frac{1}{2} H(0) C_{N p}^{p}\left(\frac{N-p}{p-1}\right)^{p} \int_{R^{N-1}}\left(1+\left|x^{\prime}\right|^{\frac{p}{p-1}}\right)^{-N}\left|x^{\prime}\right|^{\frac{3 p-2}{p-1}} \mathrm{~d} x^{\prime}=K_{1}, \\
& \lim _{\varepsilon \rightarrow 0} \varepsilon^{-\frac{p-1}{p}} K_{2}(\varepsilon)=\frac{1}{2} H(0) C_{N p}^{p^{*}} \int_{R^{N-1}}\left(1+\left|x^{\prime}\right|^{\frac{p}{p-1}}\right)^{-N}\left|x^{\prime}\right|^{2} \mathrm{~d} x^{\prime}=K_{2} .
\end{aligned}
$$

(2)

$$
\int_{\Omega}\left|u_{\varepsilon}\right|^{p} \mathrm{~d} x= \begin{cases}O\left(\varepsilon^{\frac{N-p}{p}}\right), & N<p^{2}, \\ O\left(\varepsilon^{\frac{N-p}{p}}|\ln \varepsilon|\right), & N=p^{2}, \\ O\left(\varepsilon^{p-1}\right), & N>p^{2} .\end{cases}
$$

(3)

$$
\int_{\Omega}\left|u_{\varepsilon}\right|^{q} \mathrm{~d} x= \begin{cases}O\left(\varepsilon^{\frac{q(N-p)}{p^{2}}}\right), & q<\frac{N(p-1)}{N-p}, \\ O\left(\varepsilon^{\frac{q(N-p)}{p^{2}}}|\ln \varepsilon|\right), & q=\frac{N(p-1)}{N-p}, \\ O\left(\varepsilon^{\frac{(p-1)(N p-q(N-p))}{p^{2}}}\right), & q>\frac{N(p-1)}{N-p} .\end{cases}
$$

\section{Proof of main results}

Let $\varphi(x) \in C_{0}^{\infty}\left(R^{N}\right)$ be a smooth cut off function such that

$$
\begin{aligned}
& 0 \leq \varphi(x) \leq 1, \quad \frac{\delta}{2} \leq|x| \leq \delta ; \\
& \varphi(x)=1, \quad|x|<\frac{\delta}{2} ; \\
& \varphi(x)=0, \quad|x|>\delta .
\end{aligned}
$$

Define $\omega_{\varepsilon}=\varphi u_{\varepsilon}$, then we have the following lemma about $\omega_{\varepsilon}$. 
Lemma 3.1 Suppose $N \geq 2 p, 0 \in \partial \Omega$. If the function $V(x)$ satisfies $\int_{\Omega \cap B(0, \delta)} V^{r^{\prime}} \mathrm{d} x<\infty$, then

$$
\int_{\Omega \cap B(0, \delta)} V \omega_{\varepsilon}^{p} \mathrm{~d} x=O\left(\varepsilon^{\frac{N-p}{p}+p-N+\frac{N(p-1)}{p r}}\right),
$$

where $\frac{1}{r}+\frac{1}{r^{\prime}}=1,1<r<\frac{N(p-1)}{N p+2 p-N-p^{2}-1}$.

Proof According to the Hölder inequality and the definition of $\omega_{\varepsilon}$, we have

$$
\begin{aligned}
& \int_{\Omega \cap B(0, \delta)} V \omega_{\varepsilon}^{p} \mathrm{~d} x \leq\left(\int_{\Omega \cap B(0, \delta)} V^{r^{\prime}} \mathrm{d} x\right)^{\frac{1}{r^{\prime}}}\left(\int_{\Omega \cap B(0, \delta)} \omega_{\varepsilon}^{p r} \mathrm{~d} x\right)^{\frac{1}{r}} \\
& \leq \varepsilon^{\frac{(N-p)}{p}} C_{N p}^{p}\left(\int_{\Omega \cap B(0, \delta)} V^{r^{\prime}} \mathrm{d} x\right)^{\frac{1}{r^{\prime}}}\left(\int_{B(0, \delta)}\left(\varepsilon+|x|^{\frac{p}{p-1}}\right)^{r(p-N)} \mathrm{d} x\right)^{\frac{1}{r}} \\
&= \varepsilon^{\frac{N-p}{p}+p-N+\frac{N(p-1)}{p r}} C_{N p}^{p}\left(\int_{\Omega \cap B(0, \delta)} V^{r^{\prime}} \mathrm{d} x\right)^{\frac{1}{r^{\prime}}} \\
& \times\left(\int_{B(0, \delta \varepsilon}-\frac{p-1}{p}\right) \\
&
\end{aligned}
$$

Noting that $\frac{N(p-1)}{(N-p) p} \leq 1<r$, a series of computations yield

$$
\int_{\Omega \cap B(0, \delta)} V \omega_{\varepsilon}^{p} \mathrm{~d} x=O\left(\varepsilon^{\frac{N-p}{p}+p-N+\frac{N(p-1)}{p r}}\right) .
$$

Lemma 3.2 Suppose that (A1), (A2) hold and $0 \in \partial \Omega, H(0)>0, Q_{m}=Q(0)$. If the functions $Q(x), V(x)$ satisfy the conditions (A3), (A4), then there exists a nonnegative function $v \in$ $W_{\lambda, V}^{1, p}(\Omega), v \neq \equiv$, such that

$$
\sup _{t \geq 0} J_{\lambda}(t v)<c^{*}
$$

for each $\lambda>0, N \geq 2 p$.

Proof We divide the proof into three steps.

(i) We consider the functional

$$
\begin{aligned}
g(t)= & J_{\lambda}\left(t \omega_{\varepsilon}\right) \\
= & \frac{t^{p}}{p} \int_{\Omega}\left(\left|\nabla \omega_{\varepsilon}\right|^{p}+\lambda V(x)\left|\omega_{\varepsilon}\right|^{p}\right) \mathrm{d} x-\frac{t^{p *}}{p^{*}} \lambda \int_{\Omega} Q(x)\left|\omega_{\varepsilon}\right|^{p^{*}} \mathrm{~d} x \\
& -\frac{t^{q}}{q} \lambda \int_{\Omega} P(x)\left|\omega_{\varepsilon}\right|^{q} \mathrm{~d} x, \quad t>0 .
\end{aligned}
$$

Noting that $\lim _{t \rightarrow \infty} g(t)=-\infty, g(0)=0, g(t)>0$ for $t \rightarrow 0^{+}$, we know that there exists a $t_{\varepsilon}>0$ such that $\sup _{t>0} g(t)$ is attained for $t_{\varepsilon}$ and $t_{\varepsilon}$ is uniformly bounded for $\varepsilon>0$ sufficiently small. Thus,

$$
g\left(t_{\varepsilon}\right)=\sup _{t \geq 0} J_{\lambda}\left(t \omega_{\varepsilon}\right)
$$




$$
\begin{aligned}
\leq & \sup _{t \geq 0}\left[\frac{t^{p}}{p} \int_{\Omega}\left|\nabla \omega_{\varepsilon}\right|^{p} \mathrm{~d} x-\frac{t^{p *}}{p^{*}} \lambda \int_{\Omega} Q(x)\left|\omega_{\varepsilon}\right|^{p^{*}} \mathrm{~d} x\right] \\
& +\frac{t_{\varepsilon}^{p}}{p} \int_{\Omega} \lambda V(x)\left|\omega_{\varepsilon}\right|^{p} \mathrm{~d} x-\frac{t_{\varepsilon}^{q}}{q} \lambda \int_{\Omega} P(x)\left|\omega_{\varepsilon}\right|^{q} \mathrm{~d} x \\
= & \frac{1}{N}\left[\frac{\int_{\Omega}\left|\nabla \omega_{\varepsilon}\right|^{p} \mathrm{~d} x}{\left(\lambda \int_{\Omega} Q(x)\left|\omega_{\varepsilon}\right|^{p^{*}} \mathrm{~d} x\right)^{\frac{N-p}{N}}}\right]^{\frac{N}{p}}+\frac{t_{\varepsilon}^{p}}{p} \int_{\Omega} \lambda V(x)\left|\omega_{\varepsilon}\right|^{p} \mathrm{~d} x \\
& -\frac{t_{\varepsilon}^{q}}{q} \lambda \int_{\Omega} P(x)\left|\omega_{\varepsilon}\right|^{q} \mathrm{~d} x .
\end{aligned}
$$

(ii) When $\varepsilon>0$ is sufficiently small, we have

$$
\begin{aligned}
& \int_{\Omega} Q(x)\left|\omega_{\varepsilon}\right|^{p^{*}} \mathrm{~d} x=Q_{m} \int_{\Omega}\left|u_{\varepsilon}\right|^{p^{*}} \mathrm{~d} x+o\left(\varepsilon^{\frac{p-1}{p}}\right), \\
& \int_{\Omega}\left|\nabla \omega_{\varepsilon}\right|^{p} \mathrm{~d} x \leq \int_{\Omega}\left|\nabla u_{\varepsilon}\right|^{p} \mathrm{~d} x+o\left(\varepsilon^{\frac{p-1}{p}}\right), \\
& \int_{\Omega}\left|\omega_{\varepsilon}\right|^{q} \mathrm{~d} x=\int_{\Omega}\left|u_{\varepsilon}\right|^{q} \mathrm{~d} x+o\left(\varepsilon^{\frac{p-1}{p}}\right), \\
& \int_{\Omega}\left|\omega_{\varepsilon}\right|^{p^{*}} \mathrm{~d} x=\int_{\Omega}\left|u_{\varepsilon}\right|^{p^{*}} \mathrm{~d} x+o\left(\varepsilon^{\frac{p-1}{p}}\right) .
\end{aligned}
$$

We firstly prove the first formula. Since $|Q(x)-Q(0)|=o\left(|x|^{\alpha}\right)$ for $x \rightarrow 0$, there exists a $0<\delta_{0} \leq \delta$ such that $|Q(x)-Q(0)| \leq C|x|^{\alpha}$ for $|x|<\delta_{0}$, where $C>0$ is constant. Moreover

$$
\begin{aligned}
\int_{\Omega}|Q(x)-Q(0)|\left|\omega_{\varepsilon}\right|^{p^{*}} \mathrm{~d} x \\
\leq \int_{\Omega \cap|x| \leq \delta_{0}}|Q(x)-Q(0)|\left|\omega_{\varepsilon}\right|^{p^{*}} \mathrm{~d} x+\int_{\Omega \cap|x| \geq \delta_{0}}|Q(x)-Q(0)|\left|\omega_{\varepsilon}\right|^{p^{*}} \mathrm{~d} x \\
\leq C \int_{|x| \leq \delta_{0}}|x|^{\alpha}\left|\omega_{\varepsilon}\right|^{p^{*}} \mathrm{~d} x+2 Q_{M} \int_{\Omega \cap|x| \geq \delta_{0}}\left|\omega_{\varepsilon}\right|^{p^{*}} \mathrm{~d} x \\
\leq C C_{N p}^{p^{*}} \varepsilon^{\frac{(p-1) \alpha}{p}} \int_{|x| \leq \frac{\delta_{0}}{\varepsilon^{\frac{p-1}{p}}}|x|^{\alpha}\left(1+|x|^{\frac{p}{p-1}}\right)^{-N} \mathrm{~d} x} \\
\quad+2 Q_{M} C_{N p}^{p^{*}} \varepsilon^{\frac{N}{p}} \int_{\Omega \cap|x| \geq \delta_{0}}\left(\varepsilon+|x|^{\frac{p}{p-1}}\right)^{-N} \mathrm{~d} x \\
=O\left(\varepsilon^{\frac{(p-1) \alpha}{p}}\right)+O\left(\varepsilon^{\frac{N}{p}}\right) .
\end{aligned}
$$

Since $N \geq 2 p, 1<\alpha<\frac{N}{p-1}, \int_{\Omega}\left|Q(x)-Q(0) \| \omega_{\varepsilon}\right|^{p^{*}} \mathrm{~d} x=o\left(\varepsilon^{\frac{p-1}{p}}\right)$, which implies

$$
\begin{aligned}
\int_{\Omega} & Q(x)\left|\omega_{\varepsilon}\right|^{p^{*}} \mathrm{~d} x \\
& =Q_{m} \int_{\Omega}\left|\omega_{\varepsilon}\right|^{p^{*}} \mathrm{~d} x+\int_{\Omega}(Q(x)-Q(0))\left|\omega_{\varepsilon}\right|^{p^{*}} \mathrm{~d} x \\
& =Q_{m} \int_{\Omega}\left|\omega_{\varepsilon}\right|^{p^{*}} \mathrm{~d} x+o\left(\varepsilon^{\frac{p-1}{p}}\right) .
\end{aligned}
$$

Similarly, we can evaluate the rest of formulas and omit the details here. 
(iii) $\sup _{t \geq 0} J_{\lambda}\left(t \omega_{\varepsilon}\right)<c^{*}$.

Combining (3.3) with Lemma 2.3, one has

$$
\begin{aligned}
& \int_{\Omega}\left|\nabla \omega_{\varepsilon}\right|^{p} \mathrm{~d} x \leq M_{1}\left(1-M_{1}^{-1} K_{1}(\varepsilon)+o\left(\varepsilon^{\frac{p-1}{p}}\right)\right), \\
& \int_{\Omega}\left|\omega_{\varepsilon}\right|^{p^{*}} \mathrm{~d} x=M_{2}\left(1-M_{2}^{-1} K_{2}(\varepsilon)+o\left(\varepsilon^{\frac{p-1}{p}}\right)\right),
\end{aligned}
$$

where $M_{1}=\frac{1}{2} \int_{R^{N}}\left|\nabla u_{\varepsilon}\right|^{p} \mathrm{~d} x, M_{2}=\frac{1}{2} \int_{R^{N}}\left|u_{\varepsilon}\right|^{p^{*}} \mathrm{~d} x$. Then, using (3.2), (3.3), Lemma 2.3 and Lemma 3.1, we see that

$$
\begin{aligned}
\sup _{t \geq 0} J_{\lambda}\left(t \omega_{\varepsilon}\right) \leq & \frac{S^{\frac{N}{p}}}{2 N\left(\lambda Q_{m}\right)^{\frac{N-p}{p}}}\left[1+\frac{N-p}{p} M_{2}^{-1} K_{2}(\varepsilon)-\frac{N}{p} M_{1}^{-1} K_{1}(\varepsilon)+o\left(\varepsilon^{\frac{p-1}{p}}\right)\right] \\
& +O\left(\varepsilon^{\frac{N-p}{p}+p-N+\frac{(p-1) N}{p r}}\right) .
\end{aligned}
$$

Next, we claim that

$$
\lim _{\varepsilon \rightarrow 0} \varepsilon^{\frac{p-1}{p}}\left[\frac{N-p}{p} M_{2}^{-1} K_{2}(\varepsilon)-\frac{N}{p} M_{1}^{-1} K_{1}(\varepsilon)\right]<0
$$

for $\varepsilon>0$ small enough, which implies (3.1) holds. According to $\lim _{\varepsilon \rightarrow 0} \varepsilon^{\frac{p-1}{p}} K_{1}(\varepsilon)=K_{1}$, $\lim _{\varepsilon \rightarrow 0} \varepsilon^{\frac{p-1}{p}} K_{2}(\varepsilon)=K_{2}$, we know that (3.4) is equivalent to $\frac{K_{1}}{K_{2}}>\frac{N-p}{N} \frac{M_{1}}{M_{2}}$.

From the expressions of $K_{1}, K_{2}, M_{1}, M_{2}$ and $u_{\varepsilon}$, a series of computations yield

$$
\begin{aligned}
\frac{K_{1}}{K_{2}} & =\frac{\frac{1}{2} H(0) C_{N p}^{p}\left(\frac{N-p}{p-1}\right)^{p} \int_{R^{N-1}}\left(1+\left|x^{\prime}\right|^{\frac{p}{p-1}}\right)^{-N}\left|x^{\prime}\right|^{\frac{3 p-2}{p-1}} \mathrm{~d} x^{\prime}}{\frac{1}{2} H(0) C_{N p}^{p^{*}} \int_{R^{N-1}}\left(1+\left|x^{\prime}\right|^{\frac{p}{p-1}}\right)^{-N}\left|x^{\prime}\right|^{2} \mathrm{~d} x^{\prime}} \\
& =C_{N p}^{p-p^{*}}\left(\frac{N-p}{p-1}\right)^{p} \frac{\int_{0}^{\infty}\left(1+r^{2}\right)^{-N} r \frac{2 N p+3 p-2 N-2}{p}}{\int_{0}^{\infty}\left(1+r^{2}\right)^{-N} r} \mathrm{~d} r \\
\frac{N-p}{N} \frac{M_{1}}{M_{2}} & =\frac{N-p}{N} \frac{\int_{R^{N}}\left|\nabla u_{\varepsilon}\right|^{p} \mathrm{~d} x}{\int_{R^{N}}\left|u_{\varepsilon}\right|^{*} \mathrm{~d} x} \mathrm{~d} r \\
& =\frac{N-p}{N} C_{N p}^{p-p^{*}}\left(\frac{N-p}{p-1}\right)^{p} \frac{\int_{0}^{\infty}\left(1+r^{2}\right)^{-N} r^{\frac{2 N p+p-2 N}{p}}}{\int_{0}^{\infty}\left(1+r^{2}\right)^{-N} r^{\frac{2 N p-p-2 N}{p}} \mathrm{~d} r} .
\end{aligned}
$$

Integrating by parts, we have

$$
\int_{0}^{\infty} \frac{r^{\beta}}{\left(1+r^{2}\right)^{n}} \mathrm{~d} r=\frac{\beta-1}{2 n-\beta-1} \int_{0}^{\infty} \frac{r^{\beta-2}}{\left(1+r^{2}\right)^{n}} \mathrm{~d} r \quad \text { for } 2 \leq \beta<2 n-1
$$

Then

$$
\begin{aligned}
& \frac{K_{1}}{K_{2}}=C_{N p}^{p-p^{*}}\left(\frac{N-p}{p-1}\right)^{p} \frac{(p-1)(N+1)}{N-2 p+1}, \\
& \frac{N-p}{N} \frac{M_{1}}{M_{2}}=C_{N p}^{p-p^{*}}\left(\frac{N-p}{p-1}\right)^{p}(p-1) .
\end{aligned}
$$


This implies $\frac{K_{1}}{K_{2}}>\frac{N-p}{N} \frac{M_{1}}{M_{2}}$. Thus

$$
\sup _{t \geq 0} J_{\lambda}\left(t \omega_{\varepsilon}\right)<\frac{S^{\frac{N}{p}}}{2 N\left(\lambda Q_{m}\right)^{\frac{N-p}{p}}}=c^{*} .
$$

The proof of Lemma 3.2 is complete.

Proof of Theorem 1.1 Applying Lemma 2.1 and Lemma 3.2, we obtain

$$
c=\inf _{h \in \Gamma} \max _{t \in[0,1]} J_{\lambda}(h(t)) \leq \sup _{t \geq 0} J_{\lambda}\left(t \omega_{\varepsilon}\right)<c^{*} .
$$

From Lemma 2.2 and the mountain pass theorem, we know that there exists at least one nontrivial solution to Problem (1.5). Since $J_{\lambda}(u) \geq J_{\lambda}(|u|)$, Problem (1.5) has at least one nonnegative nontrivial solution. The proof of Theorem 1.1 is complete.

Proof of Theorem 1.2 Consider the following function:

$$
\begin{aligned}
h(t)= & J_{\lambda}(t u) \\
= & \frac{t^{p}}{p} \int_{\Omega}\left(|\nabla u|^{p}+\lambda V(x)|u|^{p}\right) \mathrm{d} x-\frac{t^{p *}}{p^{*}} \lambda \int_{\Omega} Q(x)|u|^{p^{*}} \mathrm{~d} x \\
& -\frac{t^{q}}{q} \lambda \int_{\Omega} P(x)|u|^{q} \mathrm{~d} x, \quad t>0 .
\end{aligned}
$$

Since $V \in L^{1}(\Omega)$, we find that

$$
\begin{aligned}
\sup _{t \geq 0} h(t) & =\sup _{t \geq 0}\left[\frac{t^{p}}{p} \int_{\Omega} \lambda V(x)|A|^{p} \mathrm{~d} x-\frac{t^{p *}}{p^{*}} \lambda \int_{\Omega} Q(x)|A|^{p^{*}} \mathrm{~d} x-\frac{t^{q}}{q} \lambda \int_{\Omega} P(x)|A|^{q} \mathrm{~d} x\right] \\
& \leq \frac{\lambda}{N}\left[\frac{\int_{\Omega} V(x) \mathrm{d} x}{\left(\int_{\Omega} Q(x) \mathrm{d} x\right)^{\frac{N-p}{N}}}\right]^{\frac{N}{p}} \text { for } u=A .
\end{aligned}
$$

Then $\sup _{t \geq 0} J_{\lambda}(t A)<c^{*}$ for $\lambda<\frac{S\left(\int_{\Omega} Q(x) \mathrm{d} x\right)^{\frac{N-p}{N}}}{Q_{M}^{\frac{N-p}{N}} \int_{\Omega} V(x) \mathrm{d} x}$.

Similarly,

$$
\begin{aligned}
J_{\lambda}(t A) & =\sup _{t \geq 0} h(t) \\
& =\sup _{t \geq 0}\left[\frac{t^{p}}{p} \int_{\Omega} \lambda V(x)|A|^{p} \mathrm{~d} x-\frac{t^{p *}}{p^{*}} \lambda \int_{\Omega} Q(x)|A|^{p^{*}} \mathrm{~d} x-\frac{t^{q}}{q} \lambda \int_{\Omega} P(x)|A|^{q} \mathrm{~d} x\right] \\
& \leq \lambda\left(\frac{q-p}{p q}\right) \frac{\left(\int_{\Omega} V(x) \mathrm{d} x\right)^{\frac{q}{q-p}}}{\left(\int_{\Omega} P(x) \mathrm{d} x\right)^{\frac{p}{q-p}}}<c^{*}
\end{aligned}
$$

for $\lambda<\left(\frac{p q}{q-p}\right) \frac{p}{N} \frac{S}{N^{\frac{p}{N}} Q_{M}^{\frac{N-p}{N}}} \frac{\left(\int_{\Omega} P(x) \mathrm{d} x\right)^{\frac{p^{2}}{N(q-p)}}}{\left(\int_{\Omega} V(x) \mathrm{d} x\right)^{\frac{p q}{N(q-p)}}}$. 
Set

$$
\lambda_{*}=\max \left\{\frac{S\left(\int_{\Omega} Q(x) \mathrm{d} x\right)^{\frac{N-p}{N}}}{Q_{M}^{\frac{N-p}{N}} \int_{\Omega} V(x) \mathrm{d} x},\left(\frac{p q}{q-p}\right)^{\frac{p}{N}} \frac{S}{N^{\frac{p}{N}} Q_{M}^{\frac{N-p}{N}}} \frac{\left(\int_{\Omega} P(x) \mathrm{d} x\right)^{\frac{p^{2}}{N(q-p)}}}{\left(\int_{\Omega} V(x) \mathrm{d} x\right)^{\frac{p q}{N(q-p)}}}\right\},
$$

then we have $\sup _{t \geq 0} J_{\lambda}(t A)<c^{*}$ for $0<\lambda<\lambda_{*}$. Similar to the proof of Theorem 1.1, Problem (1.5) has at least one nonnegative nontrivial solution. The proof of Theorem 1.2 is complete.

Proof of Theorem 1.3 Define

$$
K=\inf _{u \in W_{0}^{1, p}\left(B\left(x_{0}, \delta\right)\right) \backslash\{0\}} \frac{\int_{B\left(x_{0}, \delta\right)}|\nabla u|^{p} \mathrm{~d} x}{\left(\int_{B\left(x_{0}, \delta\right)}|u|^{q} \mathrm{~d} x\right)^{\frac{p}{q}}} .
$$

Since $p<q<p^{*}$, as is well known, there exists a function $w \in W_{0}^{1, p}\left(B\left(x_{0}, \delta\right)\right)$ such that

$$
K=\frac{\int_{B\left(x_{0}, \delta\right)}|\nabla w|^{p} \mathrm{~d} x}{\left(\int_{B\left(x_{0}, \delta\right)}|w|^{q} \mathrm{~d} x\right)^{\frac{p}{q}}}
$$

Thus,

$$
\begin{aligned}
\sup _{t \geq 0} J_{\lambda}(t w) & \leq \sup _{t \geq 0}\left[\frac{t^{p}}{p} \int_{B\left(x_{0}, \delta\right)}\left(|\nabla w|^{p}+\lambda V(x)|w|^{p}\right) \mathrm{d} x-\frac{t^{q}}{q} \lambda \int_{B\left(x_{0}, \delta\right)} P(x)|w|^{q} \mathrm{~d} x\right] \\
& \leq \frac{q-p}{p q} \frac{\left(\int_{B\left(x_{0}, \delta\right)}|\nabla w|^{p} \mathrm{~d} x\right)^{\frac{q}{q-p}}}{P_{m}^{\frac{p}{q-p}}\left(\int_{B\left(x_{0}, \delta\right)} \lambda|w|^{q} \mathrm{~d} x\right)^{\frac{p}{q-p}}} \\
& =\frac{q-p}{p q} \frac{K^{\frac{q}{q-p}}}{\lambda^{\frac{p}{q-p}} P_{m}^{\frac{p}{q-p}}} .
\end{aligned}
$$

Let $\lambda^{*}=\left(\frac{N(q-p) K^{\frac{q}{q-p}} Q_{M}^{\frac{N-p}{p}}}{p q S^{\frac{N}{p}} P_{m}^{\frac{p}{q-p}}}\right)^{\frac{p(q-p)}{N p+p q-N q}}$, where $P_{m}=\min _{x \in B\left(x_{0}, \delta\right)} P(x)$, then $\sup _{t \geq 0} J_{\lambda}(t w)<c^{*}$ for $\lambda>\lambda^{*}$. Similar to the proof of Theorem 1.1, Problem (1.5) has at least one nonnegative nontrivial solution for $\lambda>\lambda^{*}$. The proof of Theorem 1.3 is complete.

Proof of Theorem 1.4 Fix $n \in N$, let $\varphi_{1}, \varphi_{2}, \ldots, \varphi_{n} \in C_{0}^{\infty}\left(R^{N}\right)$ be smooth functions such that $\operatorname{supp} \varphi_{j} \subset B\left(x_{0}, \delta\right), j=1,2, \ldots, n, \operatorname{supp} \varphi_{i} \cap \operatorname{supp} \varphi_{j}=\varnothing, i \neq j$.

We define $E_{n}=\operatorname{Span}\left\{\varphi_{1}, \varphi_{2}, \ldots, \varphi_{n}\right\}, \Sigma$ is the set of all symmetric and closed subsets of $W_{V}^{1, p}(\Omega), \gamma(A)$ is the Krasnoselski genus,

$$
\left.i(A)=\min _{h \in \Gamma} \gamma(h(A)) \cap \partial B_{\beta_{\lambda}}\right), \quad A \in \Sigma,
$$

where $\Gamma$ is the set of all odd homomorphisms $C^{1}\left(W_{V}^{1, p}(\Omega), W_{V}^{1, p}(\Omega)\right)$.

Set

$$
c_{j}=\inf _{i(A) \geq j} \sup _{u \in A} J_{\lambda}(u), \quad j=1,2, \ldots, n .
$$


Since $i\left(E_{n}\right)=\operatorname{dim} E_{n}=n$ and $J_{\lambda}(u) \geq \beta_{\lambda}$ for $\|u\|_{\lambda V}=\rho_{\lambda}$ in Lemma 2.1, we find that

$$
\beta_{\lambda} \leq c_{1} \leq c_{2} \leq \cdots \leq c_{n} \leq \sup _{u \in E_{n}} J_{\lambda}(u)
$$

We now estimate $\sup _{u \in E_{n}} J_{\lambda}(u)$. If $u \in E_{n}$, one has $u=\sum_{j=1}^{n} \tau_{j} \varphi_{j}$ for $\tau_{j} \in R$. From the properties of $\varphi_{j}$, we obtain

$$
\begin{aligned}
\sup _{u \in E_{n}} J_{\lambda}(u) & =\sup _{u \in E_{n}}\left(\sum_{j=1}^{n} J_{\lambda}\left(\tau_{j} \varphi_{j}\right)\right) \\
& \leq \sup _{u \in E_{n}} \sum_{j=1}^{n}\left[\frac{\tau_{j}^{p}}{p} \int_{B\left(x_{0}, \delta\right)}\left(\left|\nabla \varphi_{j}\right|^{p}+\lambda V(x)\left|\varphi_{j}\right|^{p}\right) \mathrm{d} x-\frac{\tau_{j}^{q}}{q} \lambda \int_{B\left(x_{0}, \delta\right)} P(x)\left|\varphi_{j}\right|^{q} \mathrm{~d} x\right] \\
& \leq \frac{q-p}{p q} \sum_{j=1}^{n} \frac{\left(\int_{B\left(x_{0}, \delta\right)}\left|\nabla \varphi_{j}\right|^{p} \mathrm{~d} x\right)^{\frac{q}{q-p}}}{\lambda^{\frac{p}{q-p}} P_{m}^{\frac{p}{q-p}}\left(\int_{B\left(x_{0}, \delta\right)}\left|\varphi_{j}\right|^{q} \mathrm{~d} x\right)^{\frac{p}{q-p}}} .
\end{aligned}
$$

Consequently, there exists a $\Lambda_{n}>0$ such that $\sup _{u \in E_{n}} J_{\lambda}(u)<c^{*}$ for $\lambda>\Lambda_{n}$. Similar to the proof of Theorem 1.1, Problem (1.5) has at least $n$ pairs of nonnegative nontrivial solutions. The proof of Theorem 1.4 is complete.

\section{Conclusion}

In this paper, we study the following quasilinear Neumann problem with critical Sobolev exponent:

$$
\begin{cases}-\varepsilon^{p} \Delta_{p} u+V(x)|u|^{p-2} u=Q(x)|u|^{p^{*}-2} u+P(x)|u|^{q-2} u, & x \in \Omega, \\ |\nabla u|^{p-2} \frac{\partial u}{\partial v}=0, & x \in \partial \Omega\end{cases}
$$

where the weight functions $V(x)$ is continuous in $\Omega$ and $Q(x), P(x)$ are continuous on $\bar{\Omega}$. Due to the lack of compactness of the embedding of $W^{1, p}(\Omega) \hookrightarrow L^{p^{*}}(\Omega)$ and the fact that the weight function $V(x)$ may be unbounded close to the boundary $\partial \Omega$, some classical methods may not directly be applied to our problem. We introduce a suitable weighted Sobolev space and add restrictions on the weight functions $Q(x)$ and $P(x)$ to prove the corresponding functional of problem satisfies $(P S)_{c}$-condition in a suitable range by the Lions concentration-compactness principle, then apply the mountain pass lemma, the existence and multiplicity of nontrivial solutions are obtained.

\section{Acknowledgements}

The authors would like to thank the editor and the referees for their valuable comments and suggestions, which improved the quality of our manuscript.

Funding

This work is supported by the National Natural Science Foundation of China (11601122) and by DR Fund of Henan University of Technology (150152).

\section{Abbreviations}

Not applicable 


\section{Competing interests}

The authors declare that they have no competing interests.

\section{Authors' contributions}

The authors contributed equally to the writing of this paper. All authors read and approved the final manuscript.

\section{Publisher's Note}

Springer Nature remains neutral with regard to jurisdictional claims in published maps and institutional affiliations.

Received: 18 March 2018 Accepted: 18 April 2018 Published online: 27 April 2018

\section{References}

1. Pohozaev, S.I.: Eigenfunctions of the equation $\Delta u+\lambda f(u)=0$. Sov. Math. Dokl. 5, 1408-1411 (1965)

2. Brézis, H., Nirenberg, L.: Positive solutions of nonlinear elliptic equations involving critical Sobolev exponent. Commun. Pure Appl. Math. 36, 437-477 (1983)

3. Struwe, M.: A global compactness result for elliptic boundary value problems involving limiting nonlinearities. Math. Z. 187, 511-517 (1984)

4. Ambrosetti, A., Brézis, H., Cerami, G.: Combined effects of concave and convex nonlinearities in some elliptic problems. J. Funct. Anal. 122(2), 519-543 (1994)

5. Wu, T.F.: Three positive solutions for Dirichlet problems involving critical Sobolev exponent and sign-changing. J. Differ. Equ. 249, 1549-1578 (2010)

6. Chen, J.Q., Li, S.J., Li, Y.Q.: Multiple solutions for a semilinear equation involving singular potential and critical exponent. Z. Angew. Math. Phys. 56, 453-474 (2005)

7. Ekeland, I., Ghoussoub, N.: Selected new aspects of the calculus of variations in the large. Bull. Am. Math. Soc. 39 , 207-265 (2002)

8. Comte, M., Knaap, M.C.: Existence of solutions of elliptic equations involving critical Sobolev exponents with Neumann boundary conditions in general domains. Differ. Integral Equ. 6, 1132-1146 (1991)

9. Chabrowski, J., Willem, M.: Least energy solutions of a critical Neumann problem with a weight. Calc. Var. Partial Differ. Equ. 15, 421-431 (2002)

10. Chabrowski, J., Girão, P.M.: Symmetric solutions of the Neumann problem involving a critical Sobolev exponent. Topol. Methods Nonlinear Anal. 19, 1-27 (2002)

11. Chabrowski, J.: On a critical Neumann problem with a perturbation of lower order. Acta Math. Appl. Sin. 24(3) 441-452 (2008)

12. Han, P.G., Liu, Z.X.: Positive solutions for elliptic equations involving critical Sobolev exponents and Hardy terms with Neumann boundary conditions. Nonlinear Anal. 55, 167-186 (2003)

13. Yang, H.: Positive solutions for critical inhomogeneous elliptic problems in non-contractible domains. Nonlinear Anal. 70, 952-973 (2009)

14. Garcia-Azorero, J., Peral, I., Rossi, J.: A convex-concave problem with a nonlinear boundary condition. J. Differ. Equ. $198,91-128(2004)$

15. Adimurthi, Yadava, S.: Existence of a nonradial positive solution for the critical exponent with Neumann boundary condition. J. Differ. Equ. 104, 41-68 (1995)

16. Bonanno, G., Molica Bisci, G., Radulescu, V.: Existence of three solutions for a non-homogeneous Neumann problem through Orlicz-Sobolev spaces. Nonlinear Anal. 74(14), 4785-4795 (2011)

17. Ho, K., Sim, I.: A-priori bounds and existence for solutions of weighted elliptic equations with a convection term. Adv. Nonlinear Anal. 6(4), 427-445 (2017)

18. Kristály, A., Repovš, D.: Multiple solutions for a Neumann system involving subquadratic nonlinearities. Nonlinear Anal. 74(6), 2127-2132 (2011)

19. Labropoulos, N., Radulescu, V.: On the best constants in Sobolev inequalities on the solid torus in the limit case $p=1$. Adv. Nonlinear Anal. 5(3), 261-291 (2016)

20. Pucci, P., Radulescu, V.: The impact of the mountain pass theory in nonlinear analysis: a mathematical survey. Boll. Unione Mat. Ital. 9(III), 543-582 (2010)

21. Zhang, X., Zhang, B., Repovš, D.: Existence and symmetry of solutions for critical fractional Schrödinger equations with bounded potentials. Nonlinear Anal. 142, 48-68 (2016)

22. Abreu, E.A.M., do Ó, J.M., Medeiros, E.S.: Multiplicity of positive solutions for a class of quasilinear nonhomogeneous Neumann problems. Nonlinear Anal. 60, 1443-1471 (2005)

23. Zhao, J.H., Zhao, P.H.: Existence of infinitely many weak solutions for the $p$-Laplacian with nonlinear boundary conditions. Nonlinear Anal. 69, 1343-1355 (2008)

24. Alves, C.O., Hamidi, A.E.: Nehari manifold and existence of positive solutions to a class of quasilinear problems. Nonlinear Anal. 60, 611-624 (2005)

25. Cingolani, S., Vannella, G.: Multiple positive solutions for a critical quasilinear equation via Morse theory. Ann. Inst. Henri Poincaré, Anal. Non Linéaire 26, 397-413 (2009)

26. Deng, Y.B., Peng, S.J.: Existence of multiple positive solutions for inhomogeneous Neumann problem. J. Math. Anal. Appl. 271, 155-174 (2002)

27. Guo, B., Gao, W.J.: Non-extinction of solutions to a fast diffusive $p$-Laplace equation with Neumann boundary conditions. J. Math. Anal. Appl. 422, 1527-1531 (2015)

28. Degiovanni, M., Lancelotti, S.: Linking solutions for $p$-Laplace equations with nonlinearity at critical growth. J. Funct. Anal. 256, 3643-3659 (2009)

29. Fan, H., Liu, X.: Multiple positive solutions to a class of quasi-linear elliptic equations involving critical Sobolev exponent. Monatshefte Math. 174(3), 427-447 (2014)

30. Faraci, F., Farkas, C.: A quasilinear elliptic problem involving critical Sobolev exponents. Collect. Math. 66(2), 243-259 (2015) 
31. Lee, J., Kim, Y.: Multiplicity results for nonlinear Neumann boundary value problems involving p-Laplace type operators. Bound. Value Probl. 2016, 1 (2016)

32. Guo, B., Gao, W.J.: Finite-time blow-up and extinction rates of solutions to an initial Neumann problem involving the $p(x, t)$-Laplace operator and a non-local term. Discrete Contin. Dyn. Syst. 36(2), 715-730 (2016)

33. Wang, L., Wei, Q.L., Kang, D.S.: Multiple positive solutions for $p$-Laplace elliptic equations involving concave-convex nonlinearities and a Hardy-type term. Nonlinear Anal. TMA. 74, 626-638 (2011)

34. Cirstea, F., Motreanu, D., Radulescu, V.: Weak solutions of quasilinear problems with nonlinear boundary condition. Nonlinear Anal. TMA. 43(5), 623-636 (2001)

35. D’Ambrosio, L., Mitidieri, E.: Quasilinear elliptic equations with critical potentials. Adv. Nonlinear Anal. 6(2), 147-164 (2017)

36. Klimczak, L.: Existence and multiplicity of solutions for a nonhomogeneous Neumann boundary problem. Opusc. Math. 35(6), 889-905 (2015)

37. Molica Bisci, G., Repovš, D.: Multiple solutions of $p$-biharmonic equations with Navier boundary conditions. Complex Var. Elliptic Equ. 59(2), 271-284 (2014)

38. Lions, P.L.: The concentration-compactness principle in the calculus of variations. The limit case part II. Rev. Mat. Iberoam. 1, 145-201 (1985)

\section{Submit your manuscript to a SpringerOpen ${ }^{\circ}$ journal and benefit from:}

- Convenient online submission

- Rigorous peer review

- Open access: articles freely available online

- High visibility within the field

- Retaining the copyright to your article

Submit your next manuscript at $\gg$ springeropen.com 